\title{
RESEARCH ON INTEGRATED STATIC AND DYNAMIC GEOMETRIC CALIBRATION TECHNOLOGY FOR OPTICAL SURVEYING AND MAPPING SATELLITES
}

\author{
Yi Wang ${ }^{1}$, Xin $\mathrm{Hu}^{2} *$, Guojun Xie ${ }^{1}$ \\ ${ }^{1}$ Xi'an Geovis Spatial Data Technology Co., Ltd, Xi'an, China - (wangyi, xiegj)@geovis.com.cn \\ ${ }^{2}$ Xi'an Institute of Surveying and Mapping, Xi'an, China - huxin327@163.com
}

KEY WORDS: Slow Drift, Low Frequency Error, System Error, Static Calibration, Dynamic Calibration, Positioning Without Control Points

\begin{abstract}
:
As the satellite latitude and camera temperature changes, temperature distortion in orbit makes the optical axis of the star sensor drifts slowly in the satellite body coordinate system, and the attitude measurement system contains low frequency errors that cannot be ignored. Low frequency errors are systematic in short periods, and occasional in long periods, which cause an error of a few minutes, so as to greatly reduce the positioning accuracy without control points of satellite photography. Traditional geometric calibration can only eliminate system errors caused by changes in optics in sensor platform and mechanical structures and etc., and cannot reduce the effect of low-frequency errors. An integrated static and dynamic geometric calibration method based on optical surveying and mapping satellites is proposed in this paper. The attitude system error and low frequency error are effectively detected by establishing a correction model of low frequency errors and analyzing the effective calibration process of traditional on-orbit calibration and dynamic calibration. Finally, relevant experiments are performed with image from satelliteTIANHUI-1 in seven test fields, which verifies that this low-frequency error compensation method is correct and effective.
\end{abstract}

\section{INTRODUCTION}

On-orbit geometric calibration of optical satellite can eliminate system errors caused by changes of optics in sensor platform and mechanical structures and etc., and reduce the geometric distortion of the image, which is the key step to achieve photogrammetry without ground control points. There are many research and studies on geometric calibration technology at home and abroad and experiments are also carried out for high resolution satellites by scholars and institutions. Largescale geometric calibration sites have been set up for satellites SPOT-5, IKONOS, ALOS, and Worldview. And at fixed intervals, strict systematic geometric calibration is carried out for those satellites (Bresnahan et al., 2015; Cutler, 2014; Ichitsubo et al., 2003, Jung et al., 2007) .

However, after in-orbit geometric calibration of the satellite and through the test of positioning accuracy in other regions around the world, there is still a significant error in the horizontal and elevation accuracy of the satellite image. This error is systematic in a short period, and occasional in a long period, which is a kind of low-frequency error that cannot be ignored in satellite attitude measurement system (Wang et al., 2015,2016 ). The low frequency error is related to the satellite latitude and the camera's temperature during photography. As the satellite moves in orbit, the temperature distortion causes the optical axis of the star sensor to drift slowly in the satellite body coordinate system (Guan, 2018, Pambo et al., 2017, 2018). In satellite photogrammetry with ground control points, it is possible to use the ground control points to control the effect of low-frequency errors of attitude measurement system. However, in satellite photogrammetry without ground-control points, low-frequency error must be processed with some technology.
Developed countries control the low frequency errors in attitude measurement systems through the design of star sensors(Blarre et al., 2006; Schmidt et al., 2008). The Pleiades $1 \mathrm{~A}$ satellite with $0.7 \mathrm{~m}$ resolution was launched by France in 2011. Through the combination of the SED36 star sensor and fiber optic gyro on board, its attitude determination accuracy can achieve $1.5^{\prime \prime}(1 \sigma$, the following are all $1 \sigma)$, and the positioning accuracy achieves $5.6 \mathrm{~m}$ (Amberg et al., 2013). When the SPOT series satellites SPOT6 / 7 are equipped with HYDRA star sensors, the attitude determination accuracy achieves 2", and the plane / elevation accuracy is $7.8 \mathrm{~m} / 2.5 \mathrm{~m}$ respectively (Cutler, 2014). The Worldview-3 / 4 satellites, which were launched by the American Digital Global in 2014/2016, are the commercial remote sensing satellite with highest resolution in the world. Its spatial resolution can achieve $0.31 \mathrm{~m}$, and with the attitude measurement and processing by its payload Ball CT-602, the attitude determination accuracy can achieve $0.7^{\prime \prime}$, and the plane / elevation accuracy is $2.7 \mathrm{~m} / 1.6 \mathrm{~m}$ relatively, which meets the requirement of 1: 5000 mapping.(Bresnahan et al., 2015; Hu et al., 2016; Longbotham et al., 2015).

In addition to reduce the impact on low-frequency attitude errors through satellite and payload structure design, many scholars have suggested to compensate for low-frequency errors by establishing global calibration fields. The CNES has established 21 global geometric calibration fields to classify erEErors that affect positioning accuracy as system errors, lowfrequency errors, and random errors and etc. by distributed calibration method. Then different calibration models and workflow are formed according to different characteristics of the errors, making the global plane / elevation accuracy of SPOT5 without ground control points is better than $16 \mathrm{~m} / 15 \mathrm{~m}$ relatively (Valorge, 2004). American Space Imaging has established calibration fields in Phoenix, San Diego, West

\footnotetext{
* Corresponding author
} 
Texas, and Australia, making plane / elevation accuracy of IKONONS is better than $12 \mathrm{~m} / 10 \mathrm{~m}$ without ground control points (Jacke, 2005).

The accuracy of spaceborne star sensor in China is generally lower. In the engineering practice of the TIANHUI-1 satellite, some scholars (Wang et al., 2012) analyzed the long-term star sensor data using the angle offset method and found that there are obvious periodic changes between the angles of the optical axes of star sensors, which are closely related to the position of the sun. The magnitude of the change is $60^{\prime \prime}$,and itseffect cannot be ignored. Furthermore, there are only few calibration fields in China for the satellite TIANHUI-1, so it difficult for satellite positioning without ground control points. Guan Zhichao et al. used the long-term piecewise calibration method to construct an attitude change model based on multi-order Fourier series according to the time-varying law of lowfrequency attitude errors over a long period of time. 11 orbits of long strip data of ZY-02 satellite were used to verify that the modeling accuracy of the southern hemisphere along the orbit can achieve 3 pixels (Guan, 2018), but this method used Google imagery as the control data, and the accuracy of some regions would be inconsistent. Wang Renxiang was the first to survey and map topographic map with scale of 1:50000 from TIANHUI-1 with lower attitude stability and star sensor accuracy without ground control points using Equivalent Frame Photo Bundle Adjustment method of the satellite Three-LineArray image and eliminating low-frequency errors by vertical parallax(Wang et al., 2016). An integrated static and dynamic geometric calibration method based on optical surveying and mapping satellites is proposed in this paper. The attitude system error and low frequency error are effectively detected by establishing a correction model of low frequency errors and analysing the effective calibration process of traditional onorbit calibration and dynamic calibration. Finally, relevant experiments are performed with image from satellite TIANHUI-1 in seven test fields, which improves the accuracy of satellite positioning without control points and verifies that this low-frequency error compensation method is correct and effective.

\section{ALGORITHMS}

\subsection{Mathematical Models of Three-Line-Array CCD Image Photogrammetry}

As the first generation of transmission stereoscopic surveying and mapping satellite in China, the photogrammetry goal of TIANHUI-1 is to survey and map topographic map with scale of 1: 50,000 without ground control points(Wang, 2018).The Three-Linear-Array CCD camera carried by TIANHUI-1 satellite is a kind of push-broom imaging camera, which performs three dimensional imaging functions by three photoelectric scanning imaging systems arranged with a certain spatial relationship. The Three-Line-Array CCD camera use three lens and its light structure is shown in Figure 1. The Three-Line Array of three-lens constitutes an image plane which is equivalent to a same frame. In the mechanical structure, three rays of light are intersected in one point in the image coordinate direction $x$, so that the non-intersect caused by mechanical constraints remains only in the direction $y$.Three CCD lines arrays are parallel in space.

In theory, the angle between front camera and bottom camera and the angle between back camera and bottom camera are both $\alpha$, as the follow:

$$
\alpha_{l}=\alpha_{r}=\alpha
$$

Focal length of the bottom camera:

$$
F_{v}=f_{v}=f
$$

Focal length of front camera and back camera:

$$
F_{1}=F_{r}=f / \cos \alpha
$$

pixel number from the end to the main point in CCD line array in direction $y$.

$$
y_{c c d}=l / 2
$$

Where

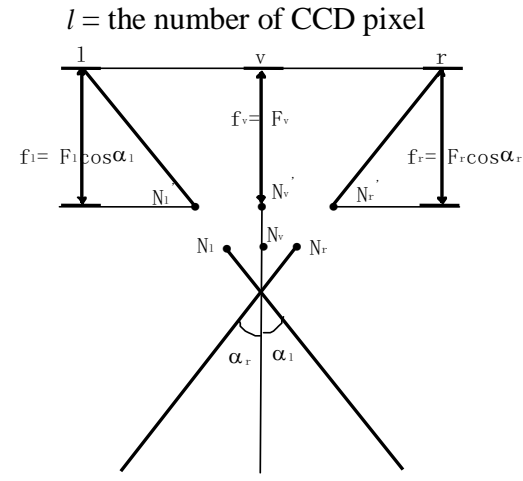

Figure 1. Three-Line-Array CCD camera of TIANHUI-1 satellite

Each scan line in the line array sensor image corresponds to a set of orbit and attitude parameters. By establishing a mathematical model of the object space and image space of the Three-Line-Array stereo image pair, the photographic beam of the Three-Line-Array CCD camera in orbit can be restored to achieve precise positioning of target point. A series of coordinate system transformations and the strict geometric relationship from the object-to-image imaging of the aerospace remote sensing satellite is:

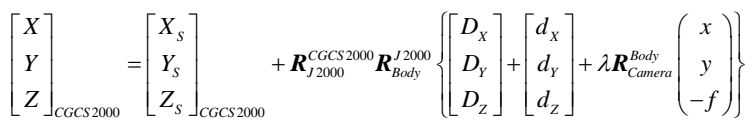

Where $\left[\begin{array}{ll}X & Y\end{array}\right]^{T} \mathrm{Z}=$ coordinates of ground point in CGCS2000 coordinate system

$\left[\begin{array}{lll}X_{S} & Y_{S} & Z_{S}\end{array}\right]^{T}=$ coordinates of ground point in CGCS2000 coordinate system

$\boldsymbol{R}_{J 2000}^{\text {WGS4 }}=$ the transformation matrix from $\mathbf{J} 2000$ coordinate system to CGCS2000 coordinate system

$\boldsymbol{R}_{\text {Body }}^{J 2000}=$ the transformation matrix from body coordinate system to $\mathbf{J} 2000$ coordinate system

$\boldsymbol{R}_{\text {Camera }}^{\text {Body }}=$ the transformation matrix from sensor coordinate system to body coordinate system

$\left[\begin{array}{lll}D_{X} & D_{Y} & D_{Z}\end{array}\right]^{T}=$ relatively the space offset of GPS antenna phase center

$\left[\begin{array}{lll}d_{X} & d_{Y} & d_{Z}\end{array}\right]^{T}=$ lens center of sensor from the satellit

$\lambda=$ proportionality coefficient

$\left[\begin{array}{lll}x & y & -f\end{array}\right]^{T}=$ coordinate of image point $\mathrm{P}$ in sensor coordinate system

$f=$ principle distance of camera 


\subsection{Static Geometric Calibration Technology}

After a satellite is launched into orbit, the optical components and mechanical structure of the sensor system may be changed or degraded due to the influence of launch vibration, solar radiation in orbit, and the impact of cosmic particles, which seriously affects the positioning accuracy of the target point (Wang, et al., 2014, Zhang, et al., 2019). In this part, a calibration model of the satellite camera included angle change value and the camera's interior elements is constructed. The ground control points are used to calibrate the system error of the camera's interior and exterior parameters generally every six months, also known as static geometric calibration.

1. Geometric calibration of the included angle change of the Star camera and the Three-Line

According to formula (5), $\left[\begin{array}{lll}D_{X} & D_{Y} & D_{Z}\end{array}\right]^{T},\left[\begin{array}{lll}d_{X} & d_{Y} & d_{Z}\end{array}\right]^{T}$ and $R_{\text {Camera }}^{\text {Body }}$ reflect the fixed relations of orbit determination, attitude determination device and camera in satellite body coordinate system. $\left[\begin{array}{lll}D_{X} & D_{Y} & D_{Z}\end{array}\right]^{T},\left[\begin{array}{lll}d_{X} & d_{Y} & d_{Z}\end{array}\right]^{T}$ have almost consistent effect on geometric positioning accuracy and can be combined to equivalent offset value $\left[\begin{array}{lll}\Delta X & \Delta Y & \Delta Z\end{array}\right]^{T}$. The equivalent offset after combination has consistent effect with that of $R_{\text {Comera }}^{\text {Body }}$ on the ground positioning accuracy in small view field. In practical satellite operation, the above errors can be shown in satellite camera matrix $R_{\text {Camera }}^{\text {Body }}$ by adding offset matrix $R_{U}$ to compensate all errors.

$$
\left[\begin{array}{c}
X \\
Y \\
Z
\end{array}\right]_{C G C S 2000}=\left[\begin{array}{c}
X_{S} \\
Y_{S} \\
Z_{S}
\end{array}\right]_{C G C S 2000}+m \mathbf{R}_{J 2000}^{C G C S 2000} \mathbf{R}_{\text {Star }}^{J 2000} \mathbf{R}_{\text {Camera }}^{\text {Star }} \mathbf{R}_{U}\left[\begin{array}{c}
x \\
y \\
-f
\end{array}\right]
$$

Offset matrix $\boldsymbol{R}_{U}$ consists of three offset angles as pitch angle deviation $\Delta \varphi$, roll angle deviation $\Delta \omega$ and yaw angle deviation $\Delta \kappa$ with the reverse order $Y-X-Z$.

transform the object space to image space:

$$
\left[\begin{array}{c}
x \\
y \\
-f
\end{array}\right]=\frac{1}{m} \mathbf{R}_{C G C S 2000}^{\text {Camera }} \mathbf{R}_{U}^{T}\left[\begin{array}{c}
X-X_{S} \\
Y-Y_{S} \\
Z-Z_{S}
\end{array}\right]_{C G C S 2000}
$$

As $\mathbf{R}_{C G C S 2000}^{\text {Camera }}$ is constant, according to the Taylor series, only the three offset angles of $\boldsymbol{R}_{U}$ are needed to derive to obtain error formula:

$$
\mathbf{V}=\mathbf{A X}-\mathbf{L}
$$

In the formula, $\mathbf{A}$ is the first-order Taylor expansion coefficient corresponding to the three angles of the offset matrix, $\mathbf{X}$ is the correction value $\left(\begin{array}{llll}\Delta \varphi & \Delta \omega & \Delta \kappa\end{array}\right)$ corresponding to the three angles of the offset matrix, the initial value is set to zero, $\mathbf{L}$ is a constant, that is, the difference between the measured value and the calculated value of the image point. $\mathbf{V}$ is the error . According to least squares principle, the offset angles $\left(\begin{array}{lll}\Delta \varphi & \Delta \omega & \Delta \kappa\end{array}\right)$ are iteratively calculated.

As the camera in TIANHUI-1 satellite uses Three-Line-Array mode to image, in the calibration process, the front, bottom, and back view conditions are used to jointly establish an exterior calibration error equation solution, so that the three beams intersect in one point, and the fixed relations among
Three-Line-Array is better used to improve redundant observations.

2. In-orbit calibration of camera interior orientation elements

The calibration of the camera optical system mainly includes calibration of the lens principal point and the focal length error. According to the structure characteristics of the Three-LineArray camera, the calibration parameters of the optical system mainly include $F_{v}, F_{l}, F_{r}, y_{c c d o l}, y_{c c d 0 v}, y_{c c d o r}, \alpha_{l}, \alpha_{r}$. Among them, the focal lengths of the front, bottom, and back view cameras are converted to the main distance $\left(f_{l}, f_{v}, f_{r}\right)$, and the included angles of the front, back, and bottom view cameras are converted to the main point coordinates $\left(x_{t}, x_{v}, x_{r}\right)$. Then the interior orientation elements that needed to be calibrated of three-line camera include the main distance $\left(f_{l}, f_{v}, f_{r}\right)$ and main point coordinates $\left(x_{l}, x_{v}, x_{r}, y_{l}, y_{v}, y_{r}\right)$.

The Three-Line-Array and three lenses are equivalent to three frame cameras. There is no strict fixed relation among the interior orientation elements of each camera after equivalent transformation. Therefore, the error equations are listed separately for the three cameras:

$$
V_{i}=B t_{i}-L_{i}
$$

In the formula: $\mathrm{i}=0,1,2$, which respectively represent the front view, bottom view, and back view cameras, $t_{i}$ is the correction number of the interior orientation elements of the camera, $L_{i}$ is constant, that is, the difference between measured value and calculated value of the image point. According to the least squares principle, the correction values of interior orientation elements of the front, the bottom, and the back camera can be iteratively calculated.

As there is strong correlation between the interior and exterior parameters, if they are combined to solve, the normal equation will be seriously abnormal or even singular, making the least square estimation solution inaccurate or even impossible to solve. Therefore, to avoid this problem, a step-by-step solution strategy, that is, iteratively solves the interior and exterior calibration parameters step by step, is used in this paper to obtain the final calibration result.

\subsection{Dynamic Geometric Calibration Technology}

In photogrammetry, the geometric meaning of the parallax represents the difference between the corresponding image points in the stereo image pair. As shown in Figure.2, $S_{1}-X_{1} Y_{1} Z_{1}$ shows the photogrammetric coordinate system which sets the left camera $S_{1}$ as the origin, and $S_{2}-X_{2} Y_{2} Z_{2}$ shows the photogrammetry coordinate system which set the right camera $S_{2}$ as the origin, and its three axes are parallel with $S_{1}-X_{1} Y_{1} Z_{1} . a_{1}, a_{2}$ represent corresponding image points in the stereo image pair, the corresponding lights $S_{1} a_{1}, S_{2} a_{2}$ intersect the object points $A_{1}, A_{2}$ relatively. $N_{1}, N_{2}$ represent the projection coefficient, and $b_{x}, b_{y}, b_{z}$ represent the component of the baseline in three axes.

The parallax $Q$ here can be calculated as:

$$
Q=N_{1} Y_{1}-N_{2} Y_{2}-b_{y}
$$


In forward intersection, if the corresponding lights intersect in one point, as $A_{1}=A_{2}$, then $Q=0$, if the corresponding lights are not intersected in one point, then $Q \neq 0$.

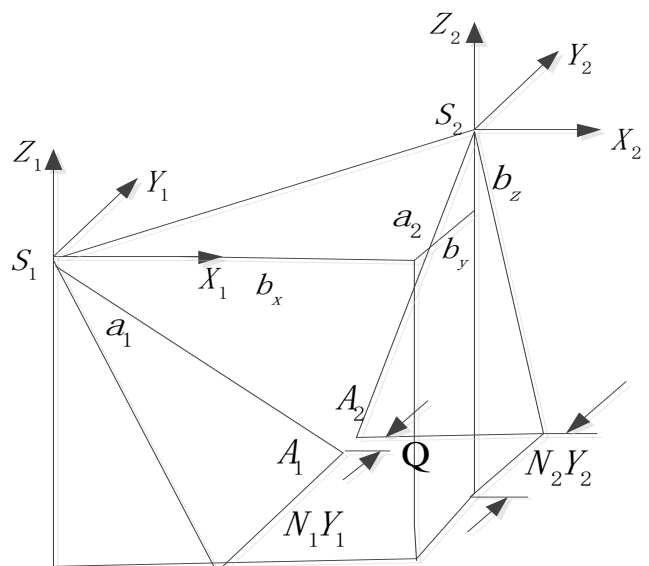

Figure 2. The geometric meaning of parallax

It is found that when the pitch and yaw angles of the image change systematically, the corresponding lights of the stereo image could not intersect together, and then there are different levels of parallax. The low frequency detection model is established using relative orientation principle, and the low frequency attitude error is obtained by eliminating parallax. Finally, the satellite attitude is compensated to obtain more accurate exterior orientation elements to improve the positioning accuracy. The entire process is without ground control points, and can be automated, which is called dynamic geometric calibration. The specific principle is as follows:

As shown in Figure.3, $a 1$ and $a 2$ are a pair of corresponding lights are located in the same epipolar plane with the photographic baseline $\mathrm{B}$, which means that the three straight lines $a 1 、 a 2$ and B are coplanar. In space analytic geometry, it can be known that if three straight lines are coplanar, the mixed product of their corresponding vectors is zero, that is,

$$
\mathrm{B} \cdot(a 1 \times a 2)=0
$$

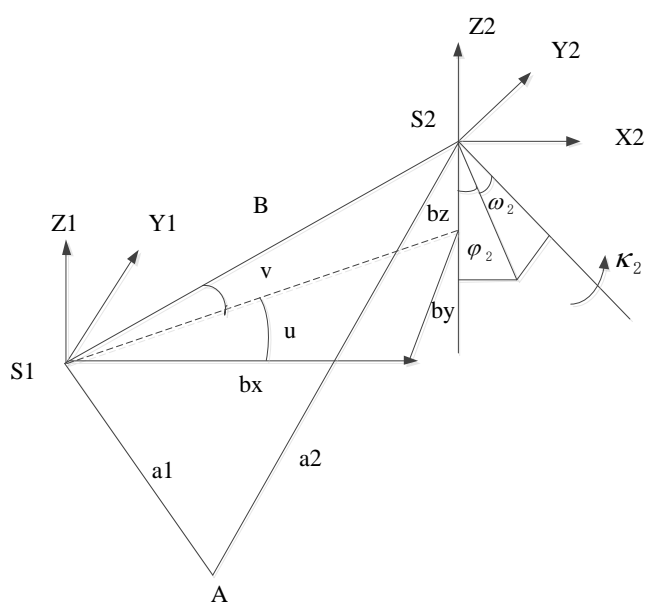

Figure 3. Relative orientation

The coordinates of the three vectors in the image space auxiliar y coordinate system are $\left(B_{x}, B_{y}, B_{z}\right),\left(X_{1}, Y_{1}, Z_{1}\right)$ and $\left(X_{2}, Y_{2}, Z_{2}\right)$ respectively. The coplanar condition equation can be expressed as coordinates:

$$
F=\left|\begin{array}{ccc}
B_{x} & B_{y} & B_{z} \\
X_{1} & Y_{1} & Z_{1} \\
X_{2} & Y_{2} & Z_{2}
\end{array}\right|=0
$$

Whether the coplanar condition equation is true or not is the criterion for completing relative orientation. To resolve relative orientation is to reslove the relative orientation element according to the coplanar condition equation.

When calculating the relative orientation, the photography baseline $\mathrm{B}$ is generally changed to $\mathrm{b}, \mathrm{b}$ is named projection baseline:

$$
B=m \cdot b
$$

In the formula, $\mathrm{m}$ is the denominator of the photographic scale; $b_{x}, b_{y}, b_{z}$ is the component of the projection baseline, it can be known from the geometric relationship in Figure 3.

$$
\left\{\begin{array}{c}
b_{y}=b_{x} \cdot \tan \mu \approx b_{x} \cdot \mu \\
b_{z}=\frac{b_{x}}{\cos \mu} \cdot \tan v \approx b_{x} \cdot v
\end{array}\right.
$$

In the above formula, $\mu$ and $v$ are the yaw angle of the baseline (that is the yaw angle of the low frequency error) and the inclination angle (that is the pitch angle of the low frequency error), it can be obtained by substituting the above formula into the coplanar condition equation:

$$
F=\left|\begin{array}{ccc}
b_{x} & b_{x} \mu & b_{x} v \\
X_{1} & Y_{1} & Z_{1} \\
X_{2} & Y_{2} & Z_{2}
\end{array}\right|=b_{x}\left|\begin{array}{ccc}
1 & \mu & v \\
X_{1} & Y_{1} & Z_{1} \\
X_{2} & Y_{2} & Z_{2}
\end{array}\right|=0
$$

The linear formula of coplanar equations is expanded according to the Taylor series, taking a small value of the first term to obtain a linear formula for coplanar equations:

$$
F=F_{0}+\frac{\partial F}{\partial \mu} d \mu+\frac{\partial F}{\partial v} d v+\frac{\partial F}{\partial \varphi} d \varphi+\frac{\partial F}{\partial \omega} d \omega+\frac{\partial F}{\partial \kappa} d \kappa=0
$$

The final derivation can be obtained,

$Q=b_{x} d \mu-\frac{Y_{2}}{Z_{2}} b_{x} d v-\frac{X_{2} Y_{2}}{Z_{2}} N_{2} d \varphi-\left(Z_{2}+\frac{Y^{2} 2}{Z_{2}}\right) N_{2} d \omega+X_{2} N_{2} d \kappa$

The error equation is set to be transferred:

$V_{Q}=b_{x} d \mu-\frac{Y_{2}}{Z_{2}} b_{x} d v-\frac{X_{2} Y_{2}}{Z_{2}} N_{2} d \varphi-\left(Z_{2}+\frac{Y_{2}^{2}}{Z_{2}}\right) N_{2} d \omega+X_{2} N_{2} d \kappa-Q$

In the equation, $X_{2}, Y_{2}, Z_{2}$ is the coordinate of corresponding image points in the right image in the photogrammetric coordinate system, $b_{x}$ is the $X$ axis component of the baseline $b, N_{2}$ is the projection coefficient of the right image, and $Q$ is the parallax. $\mu, v$ are the low frequency detection error value. The correction value of the attitude in the pitch and yaw directions are calculated by global matching of the connecting points and adjustment solution to carry out error compensation. 


\section{EXPERIMENT AND ANALYSIS}

\subsection{Experiment Data Selection}

Taking the coverage of photography into consideration, seven calibration fields in Heilongjiang Province, Xinjiang Uygur Autonomous Region, and Guangdong Province are selected according to different dimensions and conditions of uniform distribution. Those calibration fields covering different terrains such as alpine, mountainous, hilly, and flat land, with a maximum height difference of $5000 \mathrm{~m}$ can fully detect the geometric accuracy of TIANHUI-1 satellite. A strict imaging model of TIANHUI-1 satellite can be established based on the geometric and physical relationship by collecting the $1 \mathrm{~A}$ level image data of the Three-Line-Arrays corresponding to the seven calibration sites of TIANHUI-1 01 and 02 satellite.

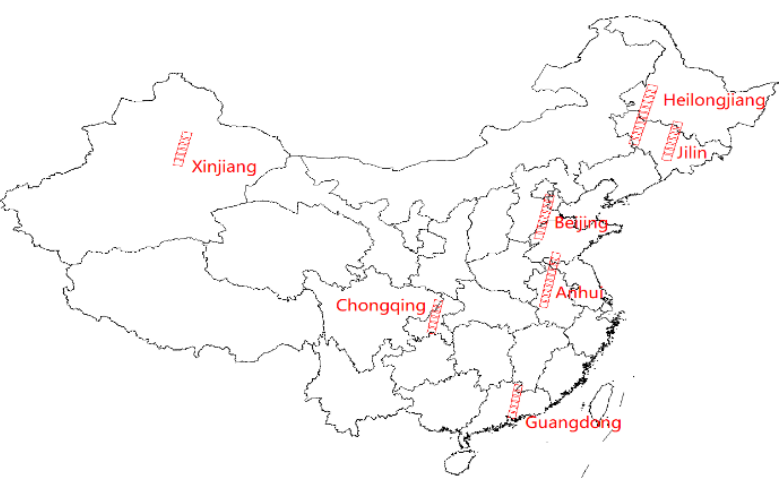

Figure 4. Distribution of seven calibration fields

\begin{tabular}{|c|c|c|c|c|c|c|c|}
\hline $\begin{array}{ll}\text { Items } & \text { Areas }\end{array}$ & Beijing & Xinjiang & Guangdong & Heilongjiang & Anhui & Jilin & Chongqing \\
\hline TH01-01 Data time & 2010.09 .23 & 2010.10 .14 & 2010.11 .11 & 2011.04 .12 & 2011.04 .24 & 2011.05 .17 & 2011.02 .24 \\
\hline TH01-02 Data time & 2013.12.11 & 2014.01 .10 & - & 2013.03.11 & 2013.04.10 & 2013.05.03 & 2013.09.16 \\
\hline \begin{tabular}{l|l} 
Range/km & Length \\
Width
\end{tabular} & $\begin{array}{c}380 \\
60\end{array}$ & $\begin{array}{c}288 \\
60\end{array}$ & $\begin{array}{c}336 \\
60\end{array}$ & $\begin{array}{c}334 \\
60\end{array}$ & $\begin{array}{c}334 \\
60\end{array}$ & $\begin{array}{c}518 \\
60\end{array}$ & $\begin{array}{c}475 \\
60\end{array}$ \\
\hline Height difference/m & 50 & 5000 & 1500 & 1300 & 1000 & 150 & 2000 \\
\hline Terrain & Flat & $\begin{array}{l}\text { high } \\
\text { mountain } \\
\text { land }\end{array}$ & $\begin{array}{l}\text { Mountain } \\
\text { land }\end{array}$ & $\begin{array}{l}\text { Mountain } \\
\text { land }\end{array}$ & hill & $\begin{array}{l}\text { Mountain } \\
\text { land }\end{array}$ & $\begin{array}{c}\text { Mountain } \\
\text { land }\end{array}$ \\
\hline
\end{tabular}

Table 1. Terrain and image data of calibration fields (Data time original:Year/month/day)

The ground control points of the calibration fields are distributed by test zone. 3-5 rows from north to south are arranged in each test zone, and 3 points in each row are evenly distributed. The number of ground control points is shown in Table 2.

\begin{tabular}{|c|c|c|c|c|c|c|c|}
\hline Test zone & Beijing & Xinjiang & Guangdong & Heilongjiang & Anhui & Jilin & Chongqing \\
\hline GCP pairs & 15 & 9 & 12 & 15 & 6 & 6 & 10 \\
\hline
\end{tabular}

Table 2. Ground control poins distribution

\subsection{Accuracy of Static Geometric Calibration}

1. Positioning accuracy analysis before and after the calibration

The interior and exterior parameters of the TH01-01 star and TH01-02 in seven calibration fields are calibrated using static geometric calibration methods, and the positioning accuracy before and after the calibration are shown in Tables 3 and 4 .

\begin{tabular}{|l|c|c|c|c|}
\hline Test zone & \multicolumn{2}{|c|}{ TH01-01 } & \multicolumn{2}{c|}{ TH01-02 } \\
\hline & $X Y$ & $H$ & $X Y$ & $H$ \\
Xinjiang & 476.20 & 12.68 & 174.31 & 41.96 \\
Beijing & 475.90 & 17.85 & 175.49 & 37.70 \\
Chongqing & 513.72 & 17.76 & 189.02 & 50.32 \\
Guangdong & 499.79 & 21.92 & - & - \\
Heilongiiang & 490.00 & 15.76 & 165.40 & 32.19 \\
Anhui & 478.88 & 23.81 & 171.80 & 52.68 \\
Jilin & 485.55 & 18.56 & 174.41 & 50.09 \\
Mean square error & 488.75 & 18.65 & 175.21 & 44.79 \\
\hline
\end{tabular}

Table 3. Postioning accuracy of seven calibration fields before the calibration $(X, Y$ : Gauss-Krueger Projection, $H$ : Geodetic height, Unit: $\mathrm{m}$ )

\begin{tabular}{|l|c|c|c|c|}
\hline Test zone & \multicolumn{2}{|c|}{ TH01-01 } & \multicolumn{2}{c|}{ TH01-02 } \\
\hline & $X Y$ & $H$ & $X Y$ & $H$ \\
Xinjiang & 7.29 & 4.87 & 9.85 & 4.9 \\
Beijing & 6.25 & 5.52 & 9.17 & 11.97 \\
Chongqing & 6.29 & 5.06 & 6.50 & 5.35 \\
Guangdong & 6.50 & 10.15 & - & - \\
Heilongjiang & 5.70 & 4.63 & 4.48 & 3.9 \\
Anhui & 3.99 & 5.38 & 6.09 & 4.14 \\
Jilin & 7.94 & 5.71 & 5.64 & 2.68 \\
Mean square error & 6.39 & 6.16 & 7.21 & 6.26 \\
\hline
\end{tabular}

Table 4. Positioning accuracy of seven calibration fields after the calibration ( $X, Y$ : Gauss-Krueger Projection, $H$ : Geodetic height, Unit: $\mathrm{m}$ )

From table 3 and table 4, we can see that:

(1) As seen in the statistical results of the positioning accuracy before calibration, there are great system errors in the original attitude data of the TH01-01 and TH01-02 satellites and the accumulated plane and elevation errors are more than hundreds of meters. And also there is great difference in plane accuracy and elevation accuracy between TH01-01 and TH0102 satellite, which means that with the different operation conditions in orbit, TH01-01 and TH01-02 satellite have different interior and exterior parameters of different cameras, so it is necessary to carry out geometric calibration and errors elimination separately for different payloads. 
(2) As seen in the statistical results of the positioning accuracy after calibration, the positioning accuracy in the plane and the elevation have been improved from hundred meters to less than ten meters, indicating that the interior and exterior parameter system errors are basically eliminated using the calibration parameters of the respective test zone.

\section{Defects in static geometric calibration}

As shown in Table 5, in less than one year of photo taking time of TH01-01 and TH01-02 satellites, the maximum change in attitude angle error is tens of seconds, which will seriously affect the subsequent positioning accuracy without ground control points.

\begin{tabular}{|l|c|c|c|c|c|c|}
\hline Test zone & \multicolumn{3}{|c|}{ TH01-01 } & \multicolumn{3}{c|}{ TH01-02 } \\
& $\Delta \varphi$ & $\Delta \omega$ & $\Delta \kappa$ & $\Delta \varphi$ & $\Delta \omega$ & $\Delta \kappa$ \\
Xinjiang & -135.6 & 112.52 & -42.37 & -40.11 & 17.91 & -105.15 \\
Beijing & -138.29 & 110.07 & -37.88 & -41.00 & 16.90 & -102.83 \\
Chongqing & -140.7 & 113.31 & -42.75 & -42.29 & 16.23 & -103.86 \\
Guangdong & -131.37 & 109.55 & -42.6 & - & - & - \\
Heilongjiang & -143.08 & 107.86 & -52.14 & -43.78 & 15.26 & -103.63 \\
Anhui & -141.39 & 107.88 & -44.68 & -41.50 & 14.15 & -100.06 \\
Jilin & -138.9 & 110.15 & -54.88 & -43.43 & 16.69 & -100.58 \\
\hline
\end{tabular}

Table 5. Statistics of calibration value of satellite camera angle (Unit: second)

The calibration parameters of the calibration fields in Beijing are selected to offset system errors for other calibration fields and carry out positioning experiment without ground control points. The statistics of positioning accuracy of each calibration fields are shown in Table 6.

\begin{tabular}{|l|c|c|c|c|}
\hline Test zone & \multicolumn{2}{|c|}{ TH01-01 } & \multicolumn{2}{c|}{ TH01-02 } \\
\hline & $X Y$ & $H$ & $X Y$ & $H$ \\
Xinjiang & 16.58 & 5.81 & 12.37 & 9.35 \\
Beijing & 7.07 & 5.92 & 9.13 & 11.97 \\
Chongqing & 18.35 & 5.63 & 12.02 & 10.43 \\
Guangdong & 16.08 & 12.15 & - & - \\
Heilongjiang & 16.88 & 6.32 & 8.89 & 7.36 \\
Anhui & 8.97 & 6.3 & 13.26 & 10.31 \\
Jilin & 18.49 & 7.28 & 10.45 & 13.38 \\
Mean square error & 15.25 & 7.38 & 11.14 & 12.3 \\
\hline
\end{tabular}

Table 6. Positioning accuracy without ground control points using calibration parameters in calibration fields of Beijing to offset that of the other fields ( $X, Y$ : Gauss-Krueger Projection, $H$ : Geodetic height, Unit: $\mathrm{m}$ )

From table 4 and table 6 , we can see that:

(1) Statistics of the mean square error in the seven calibration fields: the mean square errors in plane and elevation of the TH01-01 satellite are reduced from $6.39 \mathrm{~m} \backslash 6.16 \mathrm{~m}$ to $15.25 \mathrm{~m} \backslash 7.38 \mathrm{~m}$, and the mean square errors in plane and elevation of the TH01-02 satellite are reduced from $7.21 \mathrm{~m} \backslash$ $6.26 \mathrm{~m}$ to $11.14 \mathrm{~m} \backslash 12.3 \mathrm{~m}$;

(2) The positioning accuracy of the Beijing calibration fields has not changed, and the accuracy of other calibration fields decreased. The plane accuracy of the TH01-01 satellite has been greatly reduced, and the elevation accuracy of the TH01-02 satellite has been significantly reduced.

(3) The decrease in positioning accuracy is caused by the low frequency error of the star sensors. The period of the low frequency measurement error of the star sensor is short. The low frequency error of the star sensor occurs within a striping period (several minutes).

Taking the calibration field TH01-02 satellite in Beijing as an example, it can be seen from Table 6 that the elevation of the Beijing survey zone is $11.97 \mathrm{~m}$, which cannot meet the mapping requirements of scale 1: 50,000. The poor results of each checkpoint in the strip are analysed. As shown in Table 7, the accuracy of entire test zone is occasional. However, there is certain regularity in the change of occasional difference which means that the location of the checkpoint is closely related to the positioning accuracy. Analysis shows that the poor accuracy of checkpoints in the same row are obvious systematic. As the static geometric calibration method is based on the entire strip, so system errors in the strip cannot be detected, resulting in lower final positioning accuracy.

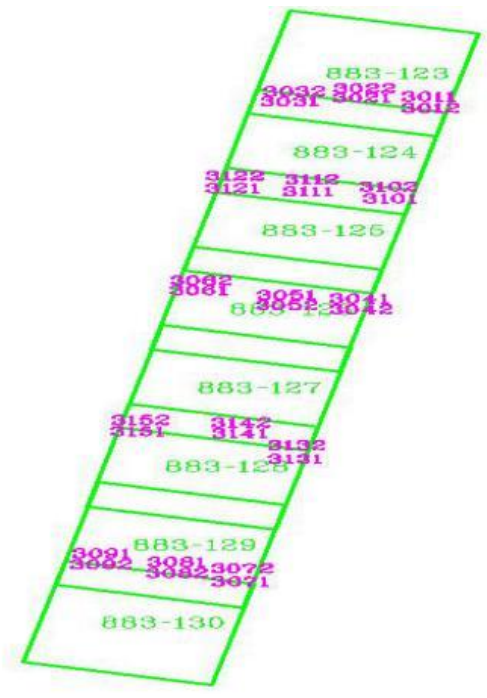

Figure 5. Distribution of ground control points and Three-LineArray images of TH01-02 in Beijing calibration fields

\begin{tabular}{|c|c|c|c|c|}
\hline $\begin{array}{c}\text { check point } \\
\text { number }\end{array}$ & $\begin{array}{c}\text { Location of } \\
\text { check points }\end{array}$ & \multicolumn{3}{|c|}{ Poor performance } \\
\hline & & $\Delta X$ & $\Delta Y$ & $\Delta H$ \\
3021 & & -10.87 & -9.45 & 1.13 \\
3022 & The first row & -1.74 & -2.09 & -6.71 \\
3031 & & -11.27 & -9.98 & -0.09 \\
3032 & & -3.25 & -4.71 & -5 \\
\hline 3111 & & 5.94 & -8.08 & 11.47 \\
3112 & & 6.68 & 0.02 & 12.27 \\
3121 & The second row & -2.74 & -4.37 & 10.77 \\
3122 & & -0.12 & -0.39 & 5.25 \\
\hline 3051 & & 14.47 & 6.42 & 15.21 \\
3052 & The third row & 14.24 & 11.38 & 19.49 \\
3061 & & 3.47 & 4.57 & 20.71 \\
3062 & & 4.14 & 1.84 & 19.08 \\
\hline 3141 & & 0.85 & -1.35 & -5.84 \\
3142 & The fourth row & 3.59 & -1.47 & -4.8 \\
3151 & & -5.96 & 7.27 & -18.57 \\
3152 & & -4.04 & 1.9 & -20.17 \\
\hline 3081 & & 0.26 & 0.6 & -5.85 \\
3082 & The fifth row & -8.26 & 5.7 & -5.55 \\
3091 & & -7.56 & -3.34 & -9.36 \\
3092 & & -10.09 & -1.05 & -2.04 \\
\hline
\end{tabular}

Table 7. Positioning accuracy without ground control points of TH01-02 in Beijing calibration fields ( $\Delta X, \Delta Y$ : GaussKrueger Projection, $\Delta H$ : Geodetic height, Unit: $\mathrm{m}$ ) 
Therefore, within a strip period, there are obvious error changes of the checkpoints. This error is systematic in a short period and random in a long period. It is a typical low frequency error of a star sensor. The static geometric calibration technology has defects that it cannot detect the change of low frequency errors.

\subsection{Offset Sensitivity Analysis of Low Frequency Error}

The system errors are basically eliminated and the low frequency measurement error should also be completely eliminated after the static geometric calibration for the seven calibration fields of TH01-01 and TH01-02 satellite. In theory, the low frequency errors of the calibration fields should be zero with the offset of Three-Line-Array image plane tilt error.

\begin{tabular}{|l|c|c|c|c|}
\hline Calibration fields & \multicolumn{2}{|c|}{$\begin{array}{c}\text { TH01-01 dynamic } \\
\text { geometric } \\
\text { calibration value }\end{array}$} & $\begin{array}{c}\text { TH01-02 dynamic } \\
\text { geometric } \\
\text { calibration value }\end{array}$ \\
\hline & $d \nu$ & $d \mu$ & $d \nu$ & $d \mu$ \\
\hline Xinjiang & 0.42 & -0.60 & -0.89 & 0.06 \\
\hline Beijing & -0.8 & 0.10 & 1.5 & -0.02 \\
\hline Chongqing & -0.25 & 0.57 & 0.54 & -0.26 \\
\hline Guangdong & -1.85 & -0.29 & - & - \\
\hline Heilongjiang & 0.99 & -0.11 & -0.13 & 0.40 \\
\hline Anhui & 0.2 & 0.52 & 1.04 & 0.17 \\
\hline Jilin & 1.31 & -0.14 & -2.06 & -0.40 \\
\hline Mean square error & 1 & 0.39 & 1.2 & 0.26 \\
\hline
\end{tabular}

Table 8 Result of dynamic geometric calibration of TH01-01 and TH01-02 satellite ( $d v$ : low frequency error of picth angle, $d \mu$ : low frequency error of yaw angle, unit: s)

The low frequency error of TH01-01 and TH01-02 satellite is detected by dynamic calibration of the angular exterior orientation element and the low frequency correction value is shown in Table 8:

(1) The pitch and yaw errors of each calibration field fluctuate around zero and are random. Through calculation mean square error, the sensitivity of low frequency error offset is about 1", and the sensitivity is relatively high;

(2) Compared with the sensitivity of pitch angle of TH01-01 and TH01-02, the sensitivity of yaw angle is higher, and it is basically zero.

\subsection{Verification of Low Frequency Error Offset}

After the dynamic calibration of calibration fields data, the low frequency error of the star sensor is corrected and positioning without ground control points are carried out. The positioning accuracy of TH01-01 and TH01-02 satellites is shown in Table 9:

\begin{tabular}{|l|c|c|c|c|}
\hline Test zone & \multicolumn{2}{|c|}{ TH01-01 } & \multicolumn{2}{c|}{ TH01-02 } \\
\hline & $X Y$ & $H$ & $X Y$ & $H$ \\
Xinjiang & 8.03 & 4.47 & 9.61 & 4.93 \\
Beijing & 6.43 & 5.52 & 10.66 & 11.99 \\
Chongqing & 8.90 & 5.07 & 7.47 & 5.30 \\
Guangdong & 8.28 & 10.15 & - & - \\
Heilongjiang & 8.92 & 4.68 & 7.63 & 3.91 \\
Anhui & 8.05 & 5.38 & 7.30 & 4.15 \\
Jilin & 6.99 & 7.08 & 9.35 & 2.69 \\
Mean square error & 7.99 & 6.32 & 8.76 & 6.27 \\
\hline
\end{tabular}

Table 9. Positioning accuracy without ground control points after low frequency error offset of TH01-01 and TH01-02 satellites $(X, Y$ : Gauss-Krueger Projection, $H$ : Geodetic height, unit: $m$ )

The positioning accuracy in Table 4 and Table 6 are analysed and compared with that in Table 9 to make the following Figures:

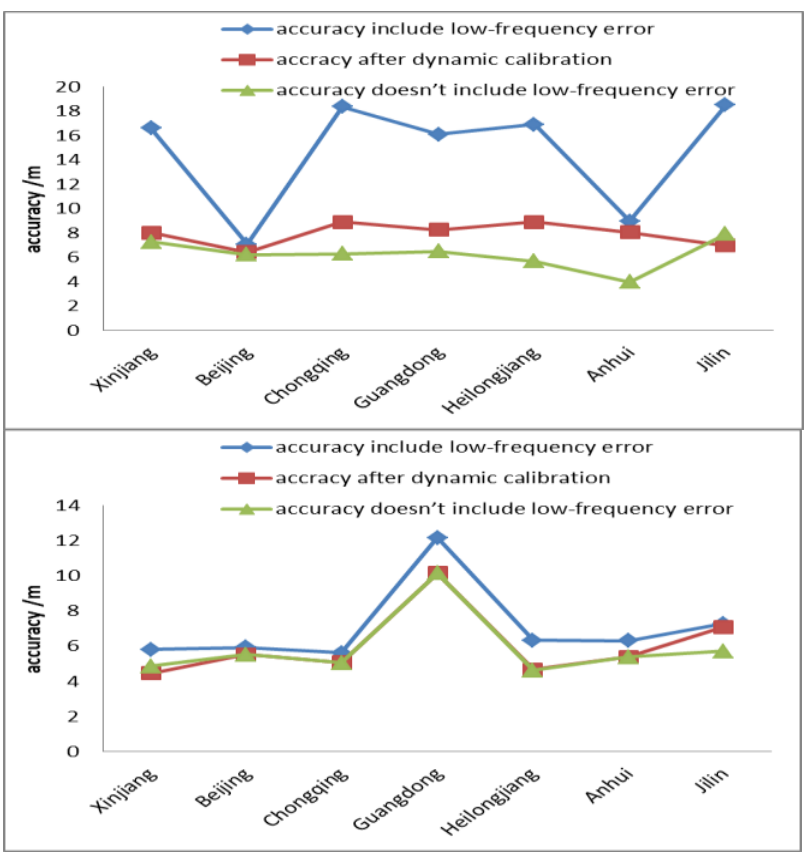

Figure 6. Plane accuracy of TH01-01 satellite and the elevation accuracy

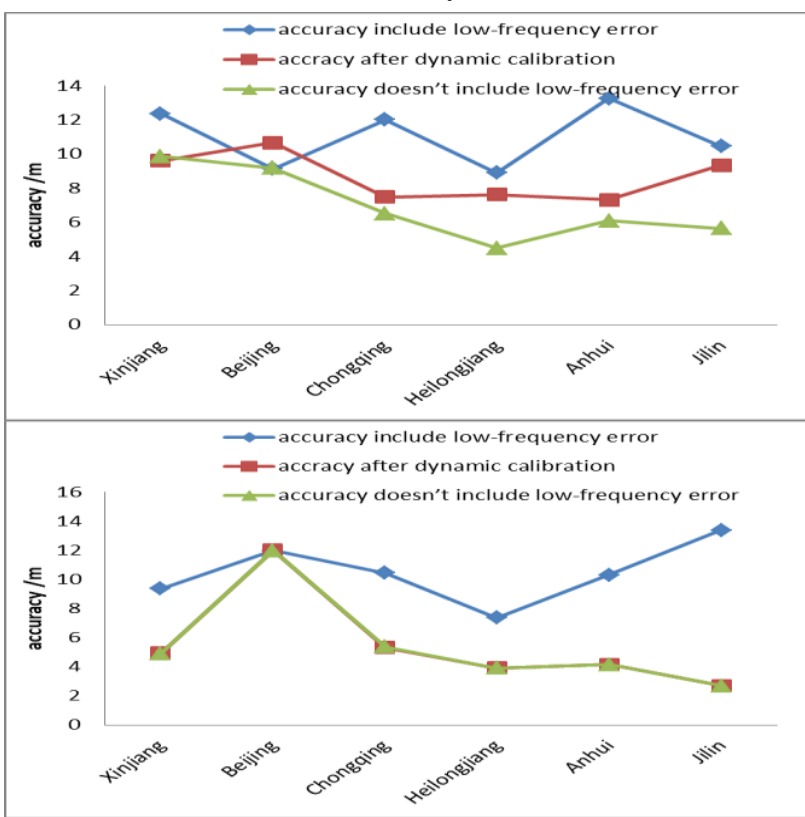

Figure 7. Plane accuracy of TH01-02 satellite and the elevation accuracy

The accuracy of each test zone is significantly lower before the dynamic geometric calibration. After dynamic geometric calibration, the plane and elevation positioning accuracy of the TH01-01 satellite is increased from $15.25 \mathrm{~m} / 7.38 \mathrm{~m}$ to $7.99 \mathrm{~m} /$ $6.32 \mathrm{~m}$, and the plane and elevation positioning accuracy TH0102 is improved from $11.14 \mathrm{~m} / 12.3 \mathrm{~m}$ to $8.76 \mathrm{~m} / 6.27 \mathrm{~m}$. 
Therefore, the integrated static and dynamic in-orbit calibration technology effectively eliminates the effect of low frequency errors. Compared with the traditional positioning results without ground control points only with static calibration, the positioning accuracy of this method is greatly improved.

\section{SUMMARY}

Based on the Three-Line-Array image data of TIANHUI-1 satellite, the characteristics of low frequency error of the star sensor and its influence on positioning accuracy are analyzed and an integrated static and dynamic geometric calibration method for optical surveying and mapping satellites is proposed. The real experiment data from seven calibration fields are used to effectively eliminate the low frequency errors in pitch angle and yaw angle of the satellite TIANHUI-1. And the positioning accuracy without ground control points is greatly improved. The plane and elevation positioning accuracy of TH01-01 satellite achieves $7.99 \mathrm{~m} / 6.32 \mathrm{~m}$, and the plane and elevation positioning accuracy of TH01-02 satellite achieves $8.76 \mathrm{~m} / 6.27 \mathrm{~m}$, which basically meets the positioning accuracy requirement of mapping scale1: 50,000 without ground control points.

\section{REFERENCE}

Amberg V., Dechoz C., Bernard L., Greslou D., Lussy F.De., Lebegue L., 2013. In-flight attitude perturbances estimation: application to PLEIADES-HR satellites, Proc. SPIE 8866(2), 206-209.

Blarre L., Ouaknine J., Oddosmarcel L., Martinez P.E., 2006. High Accuracy Sodern Star Trackers: Recent Improvements Proposed on SED36 and HYDRA Star Trackers. doi.org/10.2514/6.2006-6046.

Blarre L., Perrimon N., Airey S., 2005. New Multiple Head Star Sensor(HYDRA) Description and Status. doi.org/10.2514/6.2005-5932.

Bresnahan P., Brown E., Henryvazquez L., 2015. WorldView-3 Absolute Geolocation Accuracy Evaluation. Joint Agency Commercial Imagery Evaluation Workshop.

Cutler B., 2014. Pleiades 1B and SPOT 6 Image Quality status after commissioning and $1^{\text {st }}$ year in orbit.

Guan Z.C., 2018. Research on the Attitude Accuracy Improvement of High Resolution Pushbroom Sarellite.

Hu F., Gao X.M., Li G.Y., Li M., 2016. Dem Extraction from WorldView-3 Stereo-Images and Accuracy Evaluation. International Archives of the Photogrammetry Remote Sensing, XLI-B1, 327-332.

Ichitsubo D., 2003. Development Status for the Advanced Land Observing Satellite. International Archives of Photogrammetry, Remote Sensing and Spatial Information Science, 34(7), 14-20.

Jacke G., James L., 2005. IKONOS Geometric Calibrations. The ASPRS 2005 Annual Conference, Baltimore.

Jung H.S., Kim S.W., Won J.S., 2007. Line-of-Sight Vector Adjustment Model for Geopositioning of SPOT-5 Stereo Images. Photogrammetric Engineering and Remote Sensing, 73(11), 1267-1276.
Longbotham N.W., Pacifici F., Malitz S., Baugh, W., CampsValls G., 2015. Measuring the Spatial and Spectral Performance of WorldView-3. Hyperspectral Imaging and Sounding of the Environment, Hise 2015: HW3B.2.

Pang Bo, Li Kang, Tang Liang, Li Guo, 2017. Error Analysis and Offset Method of Star Sensors. Space Control Technology and Applcation, 43(01), 17-24.

Pang Bo, Li Kang, Tang Liang, Li Guo, 2018. A Method of inorbit Low Frequency Error Correction of Star Sensors based on Ground Landmark. Journal of Spacecraft Engineering, 27(03), 79-85.

Schmidt U., Elstner C., Michel K., 2008. ASTRO 15 Star Tracker Flight Experience and Further Improvements Towards the ASTRO APS Star Tracker. doi.org/10.2514/6.2008-6649

Toutin T., 2006. Generation of DSMs from SPOT-5 in-track HRS and across-track HRG stereo data using spatitriangulation and autocalibration. ISPRS Journal of Photogrammetry and Remote Sensing, 60(3), 170-181.

Takanori I., 2005. Precision Attitude and Position Determination for the Advanced Land Observing Satellite (ALOS). Enabling Sensor and Platform Technologies for Spaceborne Remote Sensing. 5659. doi.org/10.1117/12.579051.

Valorge C., Meygret A., Lebegue L., Henry P., Bouillon A., Gachet R., 2004. Forty years of experience with SPOT in-fligt calibration. Post-Launch calibration of Satellite Sensors. 119133.

Wang T.Y, Zhang G., Li D., Tang X., Jiang Y., Pan H., 2014. Geometric Accuracy Validation for ZY-3 Satellite Imagery. IEEE Geoscience and Remote Sensing Letters, 11(6), 11681171.

Wang R.X., Wang J.R., 2015. Discussion About the Satellite Photogrammetry without Ground Control Points. Journal of Surveying and Mapping Science, 40(02), 3-12.

Wang R.X., Wang J.R., Hu X, 2016. Low Frequency Error Offset for Satellite Photogrammetry Altitude Measurement. Journal of Surveying and Mapping, 45(02), 127-130.

Wang R.X., Wang J.R., Hu X., 2016. Initial Assessment of Positioning Accuracy of Tianhui1 03 Satellite. Journal of Surveying and Mapping, 45(10), 1135-1139.

Wang X.Y., Gao L.Y., Yi M., Wang J.R., 2012. Analysis of Positioning Errors of Stereoscopic Surveying and Mapping Satellite. Journal of Surveying and Mapping Technology and Science, 29(06), 427-429.

Wang X.T., Li Y.C., Li X.Y., 2012. Accuracy Analysis of Star Sensors of Tianhui1 Satellite. Journal of Remote Sening, 16(S1), 90-93.

Wang Y., 2018. Study on Some Key Techniques of Satellite Photogrammetry without Ground Control Points. Chang'an University, Xi'an, China.

Zhang G., Deng M.J., Cai C.L., Zhao R.S., 2019. Geometric Self-Calibration of YaoGan-13 Images Using Multiple Overlapping Images. Sensors (Basel, Switzerland), 19(10), 2367. 